\title{
Interpretation of a dilatometric anomaly previous to the ferrite-to-austenite transformation in a low carbon steel
}

\author{
T. De Cock, C. Capdevila, F.G. Caballero and C. García de Andrés \\ Solid-Solid Phase Transformation Group (Materalia) \\ Department of Physical Metallurgy, Centro Nacional de Investigaciones Metalúrgicas (CENIM), \\ Consejo Superior de Investigaciones Científicas (CSIC), Avda. Gregorio del Amo, 8, E-28040 \\ Madrid, Spain
}

Keywords: dilatometry, recrystallization, texture, ferritic steels

\begin{abstract}
During continuous heating of a cold rolled low carbon (LC) steel a dilatometric anomaly has been detected previous to the well known ferrite-to-austenite transformation. This anomaly is due to the texture change and/or the dislocation annihilation that accompany the recrystallization process.
\end{abstract}

\section{Introduction}

Dilatometry has been employed to monitor phase transformations which involve changes in lattice parameters for the last decades. However, the development of high resolution dilatometers allows to study other phenomena such as recrystallization and texture changes. Whereas the impact of texture on plastic anisotropy such as the deep drawing properties [1,2], on local orientation inhomogeneities such as ridging phenomenon in ferritic stainless steels [3,4], strength of steels [1], and elastic properties of steels [5] has already been subject to numerous investigations, its influence on the thermal expansion properties has not been treated accordingly yet. Only recently some efforts have been made to study the relation between deformation and dilatometry in extra-low carbon (ELC) steels [6] and shape-memory alloys [7].

Lattice vibrations (phonons) of the crystal lattice play a major part in the thermal expansion of the material. It is generally very sensitive to composition, planar atomic density, electron configuration and its density in the crystals, as well as to near-neighbour interactions [8-10]. These are basic reasons why two phases in an alloy system with the same crystal structure may present different thermal expansion coefficients. Since thermal expansion is reversible, similar to elastic strain, the two are interrelated.

Since texture formation (i.e., preferred orientation of lattices in the microstructure) is reported to have a significant influence on the modulus of elasticity [5] and hence should affect the thermal expansion coefficient of the material, the purpose of the present work is to analyse texture and dilatometric behaviour of a conventional low carbon steel during continuous heating when recrystallization processes take place.

\section{Materials and Experimental Techniques}

The low carbon (LC) steel sheet used in this work (0.014C-0.02Mn-0.01P) was supplied as a sheet of $0.8 \mathrm{~mm}$ in thickness prepared by hot rolled, coiled at $740{ }^{\circ} \mathrm{C}$, and a further cold rolled with reductions of $60 \%$ (sample $\mathrm{A}$ ) and $80 \%$ (sample B) at room temperature.

Texture measurements were carried out by means of the Schulz reflection method, using a D-5000 X-ray diffractometer furnished with an opened Eulerian cradle. Details of the diffractometer used and the analysis method are given elsewhere [11]. The diffraction studies were performed employing $\mathrm{Cu} \mathrm{K}_{\alpha}$ radiation and a diffracted beam monochromator. After grinding and final polishing using $0.25 \mu \mathrm{m}$ diamond paste, the samples were etched to obtain an undeformed surface. 
The pole figures (110), (200) and (211) were measured and a series expansion technique employed to calculate the orientation distribution function (ODF), along with ghost correction [12]. As both ferrite phases present a cubic crystal symmetry and the sample shape is orthorhombic, defined by rolling, normal and transverse direction ( $R D, N D, T D)$, the orientation density, $f(g)$, was represented in the reduced Bunge-Euler space $\left(0 \leq \varphi_{1}, \phi, \varphi_{2} \leq \pi / 2\right)$, see Fig 1 (a), whereas the essential texture components of LC steels are shown in Fig. 1 (b).

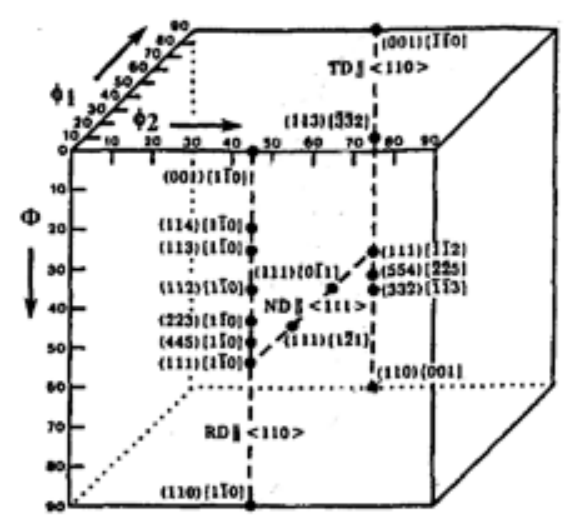

(a)

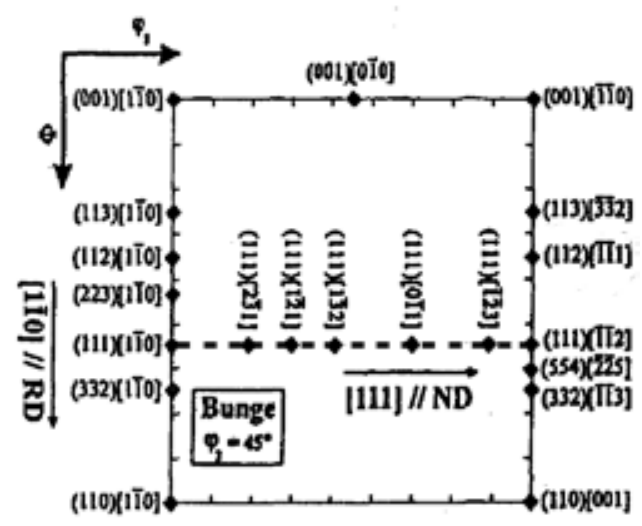

(b)

Figure 1: (a) Orientation space showing the main texture components; and (b) $\varphi_{2}=45^{\circ}$ section showing the main texture components in LC steels.

Moreover, lattice parameter measurements were conducted by X-ray analysis. The diffraction studies were performed employing $\mathrm{Cu} K \alpha$ radiation. Automatic step scanning allowed the accumulation of a sufficient number of counts at each Bragg angle to obtain the desired statistical accuracy. The lattice parameters of ferrite were calculated by a least squares method from the position of the (110), (200), (211), (220), (310) and (222) peaks. The values obtained were reproducible within $0.0001 \mathrm{~nm}$.

The dilatometric samples were mechanized as rectangular pieces of $12 \mathrm{~mm}$ length, $2 \mathrm{~mm}$ width, and $0.8 \mathrm{~mm}$ thickness. The thermal expansion of the LC steel studied has been analyzed by means of an Adamel Lhomargy DT1000 high-resolution dilatometer described elsewhere [13]. The length change of the specimen is transmitted via an amorphous silica pushrod. These variations are measured by a linear variable differential transformer (LVDT) sensor in a gas-tight enclosure enabling testing under vacuum or an inert atmosphere with an accuracy better than 0.1 micron and the dilatometric curve is monitored along the thermal cycle with the help of a computer assisted electronic device.

The thermal expansion coefficients were determined in samples heated up to $700{ }^{\circ} \mathrm{C}$ at $0.3{ }^{\circ} \mathrm{C} / \mathrm{s}$, and subsequently cooled down to room temperature at the same rate. The average thermal expansion coefficient $\left(\alpha_{\mathrm{m}}\right)$ was calculated from the dilatometric curves obtained in a temperature range between 550 and $700{ }^{\circ} \mathrm{C}$. Corrected values of $\alpha_{\mathrm{m}}$ have been obtained by considering the effect of dilatometer quartz elements on thermal expansion coefficient.

The microstructure of the as-received alloy and its evolution during recrystallization were characterized using optical microscopy (OM), and field emission scanning electron microscopy (FEG-SEM) equipped with energy dispersive X-ray microanalysis (EDX). For metallographic examination, samples were mounted in bakelite and subsequently grounded and polished according to standardized techniques. Subsequently, they were etched with $2 \%$ nital solution during $35 \mathrm{~s}-45 \mathrm{~s}$ to reveal the microstructure by optical and scanning electron microscopy, or with picral reagent to disclose the presence of cementite particles [14]. 


\section{Results and Discussion}

Texture Results

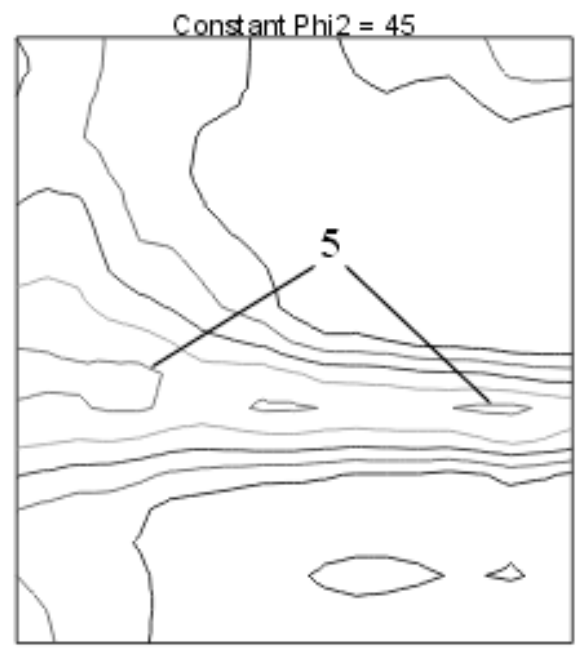

Sample A - cold rolled

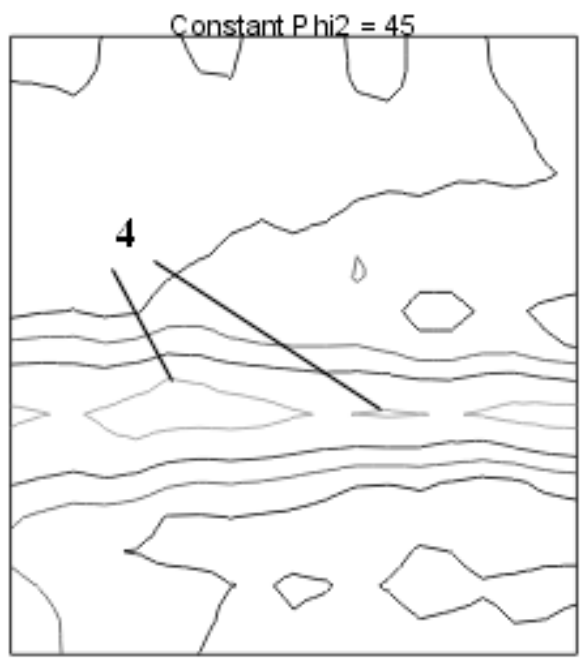

Sample A - fully annealed

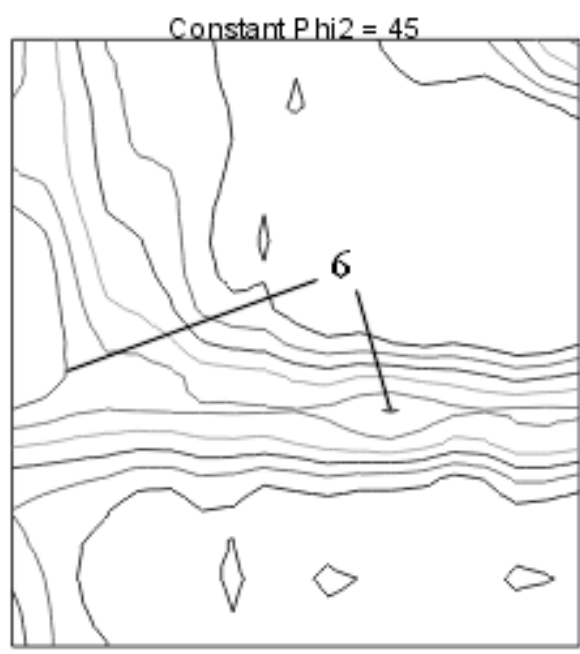

Sample B - cold rolled

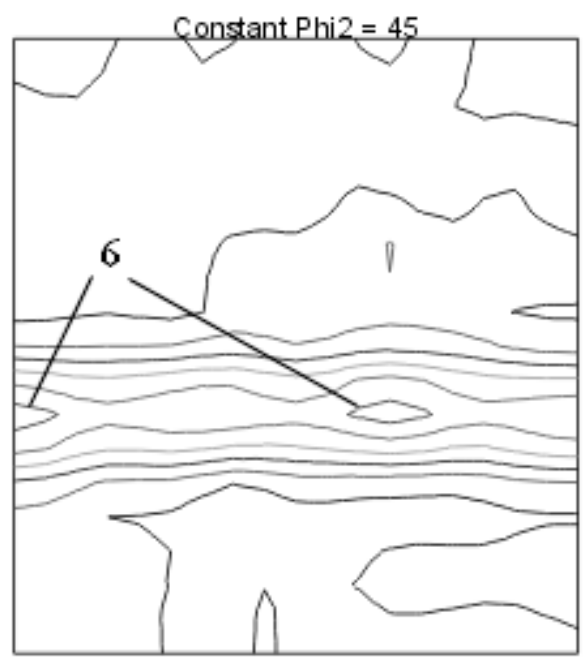

Sample B - fully annealed

Figure 2. X-ray ODFs $\left(\varphi_{2}=45^{\circ}\right)$ for the cold-rolled and fully annealed samples.

The evolution of the ODFs obtained from the X-ray measurements is presented in Fig. 2, indicating also the highest intensity obtained in each measurement. It can be seen that for the cold-rolled samples the intensity mainly concentrates along two fibers, with levels of 5 times random approximately: the $\alpha$-fiber $(\mathrm{RD} \|<110>)$, with main texture components within the range $\{001\}<110>\left(\varphi_{1}=0^{\circ}, \phi=0^{\circ}, \varphi_{2}=45^{\circ}\right)$ to $\{111\}<110>\left(\varphi_{1}=0^{\circ}, \phi=55^{\circ}, \varphi_{2}=45^{\circ}\right)$, and the $\gamma$-fiber $(\mathrm{ND} \|<111>$ ). The fully annealed samples have developed an intense $\gamma$-fiber, whereas the $\alpha$-fiber has disappeared completely, except of its intersection with the $\gamma$-fiber near the component $\{111\}<110>$. The intensity of the $\gamma$-fiber is higher in the steel with higher cold rolling reduction. These textures of the examined steel are typical for low carbon deep drawing steels [15-17].

In order to show in more detail the changes in texture in the material after recrystallization, the orientation density, $\mathrm{f}(\mathrm{g})$, along the $\alpha$ - and $\gamma$-fibers is given in Fig. 3 (a) and (b). It is clear that intensity of the $\gamma$-fiber of the as-deformed texture increases with cold rolling reduction. Likewise, recrystallization induces a decrease in the relative orientation density difference between $\{001\}<110>\left(\phi=0^{\circ}\right)$ and $\{112\}<110>\left(\phi=35^{\circ}\right)$ on the $\alpha$-fiber, whereas the component $\{111\}<110>$ 
$\left(\phi=55^{\circ}\right)$ remains practically constant. Regarding the $\gamma$-fiber in the highly reduced steel (sample B), all the components are more intense as recrystallization proceeds. In sample A this is not the case, probably due to low energy processes (leading to nuclei with orientations different from $\{111\}$ ) at lower cold rolling grades [16].

(a)

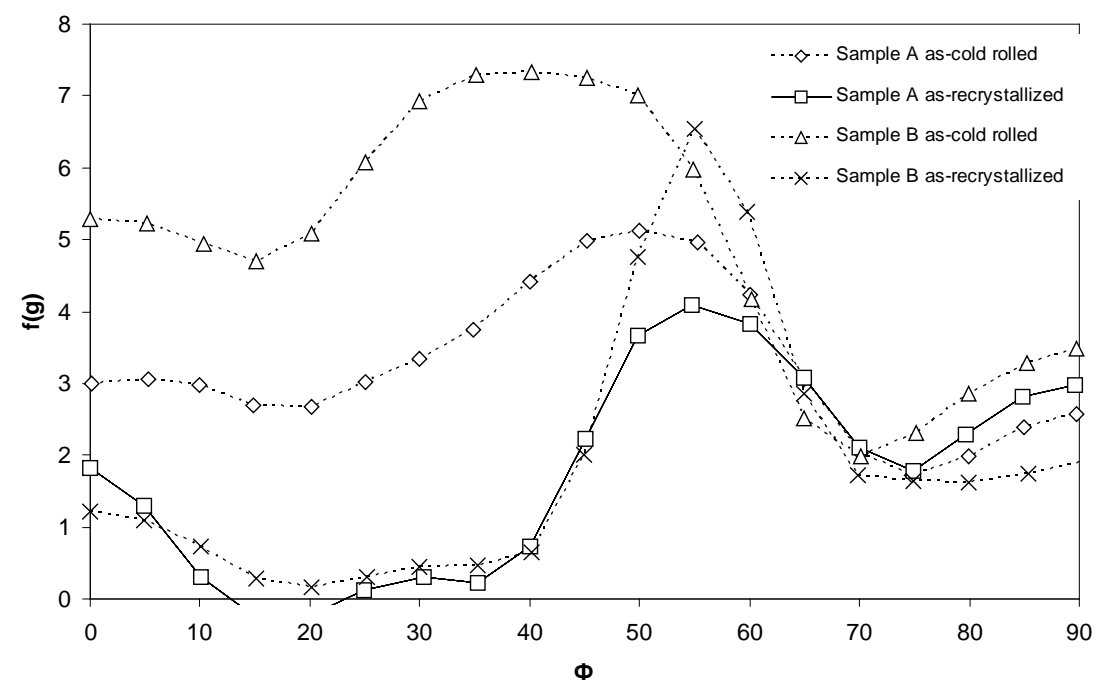

(b)

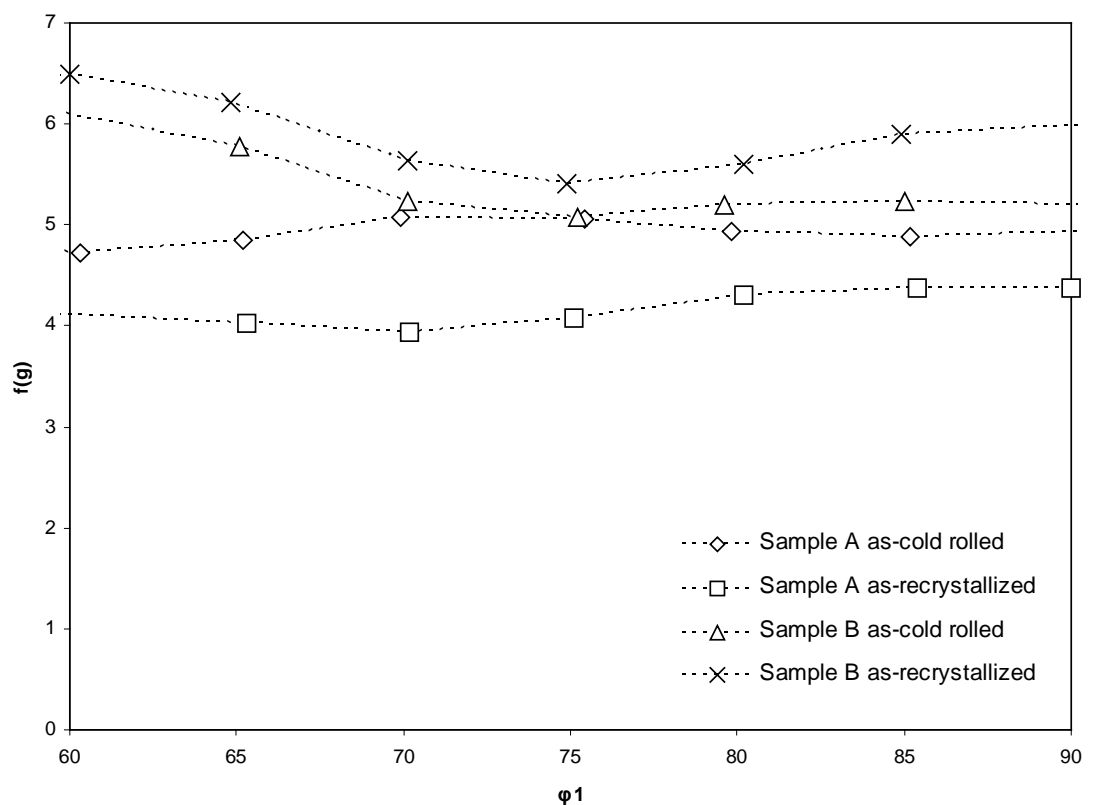

Figure 3. Evolution of the (a) $\alpha$-fiber and (b) $\gamma$-fiber for both samples.

\section{Dilatometric Results}

Fig. 4(a) shows the dilatometric curve during continuous heating of samples mechanized along rolling direction. A significant slope change is detected in both samples, which occurs well before the known dilatometric anomaly corresponding to the $\alpha \rightarrow \gamma$ transformation. For the studied steel this transformation starts at $885{ }^{\circ} \mathrm{C}$ and finishes at $919{ }^{\circ} \mathrm{C}$, whereas the temperature at which the slope change is taking place is ranging from 550 to $650{ }^{\circ} \mathrm{C}$ for both samples, as can be observed in the derivative curve in Fig. 4(b). It is the aim of the present work to clarify this slope change. 
The as-cold rolled material exhibited a microstructure with ferrite grains elongated along the rolling direction and some cementite particles (confirmed by the EDX X-ray spectra) at the grain boundaries (Fig. 5). The evolution of the microstructure during continuous heating includes two phenomena that can occur simultaneously, i.e., dissolution of carbides and recovery and recrystallization processes. The influence of cementite dissolution on thermal expansion coefficient is twofold: firstly, the local enrichment of solute elements in ferrite matrix could affect the ferrite lattice parameter; and secondly, austenite formation is promoted in the surrounding areas of carbides during its dissolution as reported in literature [18-20]. Because of the change in their specific volume values, the ferrite-to-austenite transformation induces a contraction in the dilatometric curve.

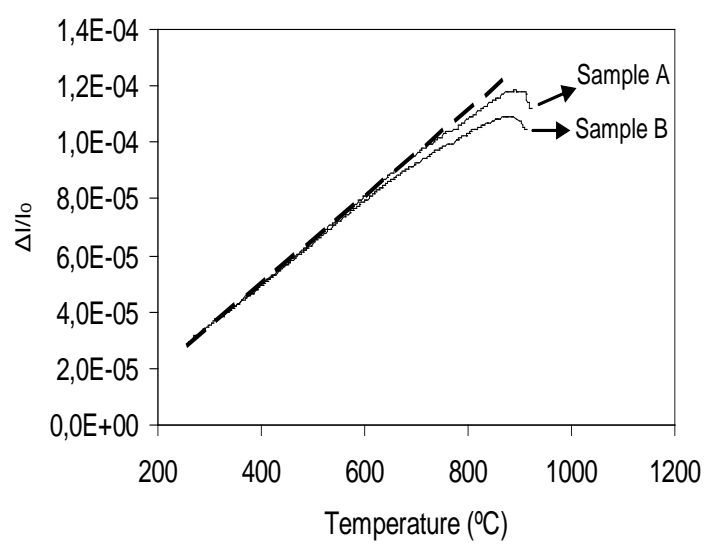

(a)

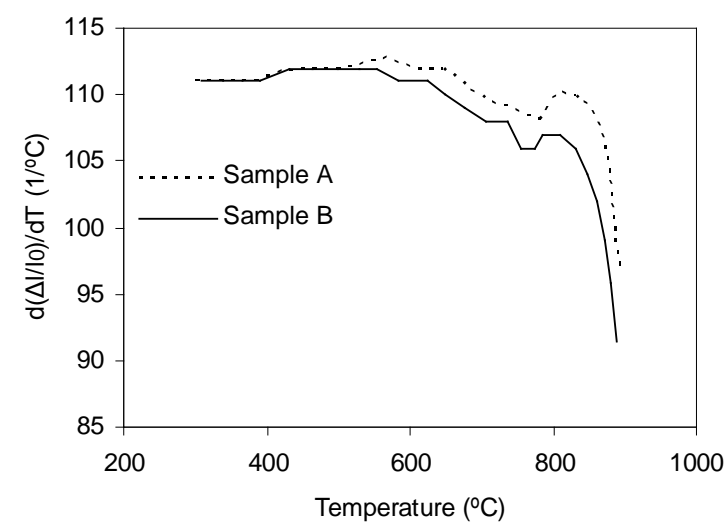

(b)

Figure 4. Dilatometric anomaly in samples A and B and (b) derivative of the dilatometric curve, taking the origin of the curves of both samples at the same point for enhanced comparison.

Interrupted heating cycles by quenching at temperatures ranging from 550 to $650{ }^{\circ} \mathrm{C}$ were carried out to find out if some dissolution of cementite has occurred. It was proved that this process is too limited to be able to sufficiently enrich in carbon surrounding areas of carbides. Hence, austenite formation around cementite particles has not occurred. Moreover, if the deviation of the dilatograms would be due to the dissolution of particles then the original slope would be almost restored after completion of the dissolution process, which is not the present case. On the other hand, X-ray analysis revealed that the broad diffraction peaks present in the diffraction pattern of the as-received sheet sharpen after annealing at temperatures higher than $650{ }^{\circ} \mathrm{C}$. This effect can be attributed to a recovery and recrystallization process. The diffraction peaks corresponding to the ferrite occupy the same position in the as-received and annealed materials. Thus, the values of the lattice parameter deduced by the least squares method did not depend on the annealing temperature, and a constant value of $2.8667 \AA$ was obtained. 


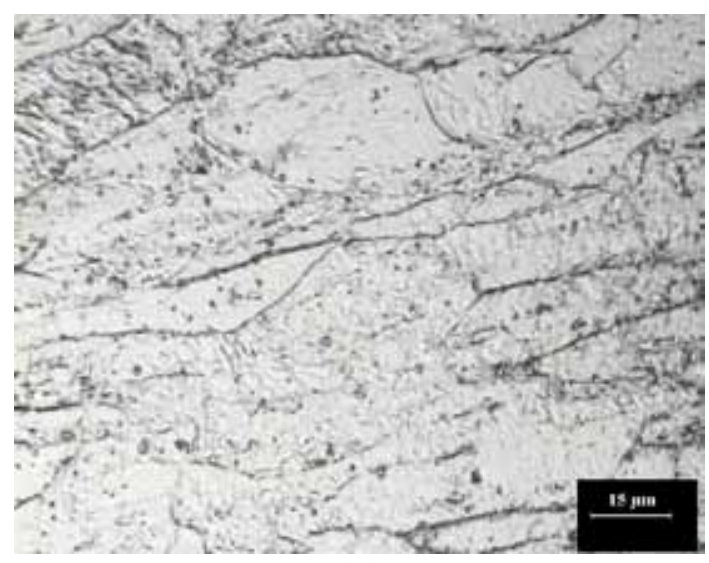

(a)

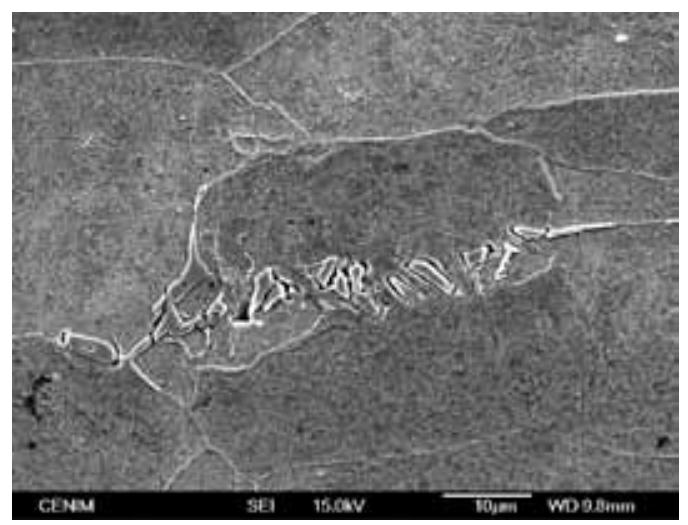

(b)

Figure 5. (a) Optical microscopy image (sample A) of the as-cold rolled microstructure, (b) FEGSEM image illustrating the presence of carbides in the grain boundary.

Measurements of the hardness evolution in the temperature range between 550 and $650{ }^{\circ} \mathrm{C}$ showed that in this temperature range recovery and recrystallization occurs. This temperature range coincides with the range in which the dilatometric anomaly is detected, as can be observed in Fig. 4 (a) and (b).

A severe cold deformation, such as is the case for the studied steel, induces a large number of dislocations in the material. The dilatation associated to dislocations are estimated to be between 1 and 2 atomic volumes for each crystal plane threaded [21-22]. The dislocation density decreases typically from $10^{16} \mathrm{~m}^{-2}$ in the as-deformed material to $10^{10} \mathrm{~m}^{-2}$ in the recrystallized sample, which is monitored with X-ray measurements and could also be detectable with a high resolution dilatometer. Thus, the relaxation of internal stresses that acompany the processes of recovery and recrystallization, i.e., rearrangement (polygonization) and annihilation of dislocations, may explain the deviation of the dilatograms.

However, since it was demonstrated that the detected anomaly also depends on whether the thermal expansion in the longitudinal or transversal direction of the samples is studied, the above explanation may not be sufficient. It is suggested that an additional and simultaneous effect to explain the slope change in the dilatometric curve, as shown in Fig. 4, is related with the change in texture produced during recrystallization. From texture analysis it was concluded that the orientations concentrate around the $\gamma$-fiber during recrystallization in detriment of the $\alpha$-fiber characteristic of as-cold rolled microstructure.

The temperature variation of the elongation strain of rolled material will be the result of two component: the elastic dilatation caused by the thermal forces and the elastic strain associated with the internal stresses via Hooke's law. The first contribution in single materials is mainly due to interatomic interactions, electronic configuration, and planar atomic density. As in cubic crystal the linear thermal expansion coefficient is isotropic, this component will be the same in a sample with and without texture. However, the second component will depend of the texture present in the material. Applying the generalized Hooke's law, the elasticity modulus of cubic crystals along different crystal directions is given as fol1ows [23, 24]

$$
1 / E_{<h k l>}=s_{11}-2\left(\left(s_{11}-s_{12}\right)-1 / 2\left(s_{44}\right)\right) L
$$

where $E_{<h k l}>$ is the directional elasticity modulus, $s_{i j}$ are the elements of the elastic compliance matrix, and $\mathrm{L}=\left(\mathrm{l}^{2} \mathrm{~h}^{2}{ }_{\mathrm{k}}+\mathrm{l}_{\mathrm{k}}^{2} \mathrm{l}^{2}{ }_{\mathrm{l}}+\mathrm{l}^{2}{ }_{\mathrm{h}} \mathrm{l}_{\mathrm{l}} \mathrm{l}\right)$, wherein $\mathrm{l}_{\mathrm{h}}$, etc., are the direction cosines of the direction with the crystal axes. Thus, the modulus in a certain direction for a cubic material is defined by its three stiffness coefficients and the particular direction, given by the direction cosine values.

As Eq. (1) indicates, the maximum value of $E$ will be obtained along the $<111>$ direction $(\mathrm{L}=\mathrm{l} / 3)$, then along the $<110>$ direction $(L=1 / 4)$, and the lowest value will be along the $<100>(L=0)$ 
direction of the bcc crystals. It should be noted that the highest $\mathrm{E}$ value (and the lowest elastic strain for a specified elastic stress) corresponds to the direction normal to the highest densely packed plane, $\{110\}$, and, likewise, the lowest value of $E$ (and the highest strain for the given elastic stress) corresponds to the direction normal to the lowest densely packed planes $\{100\}$. Therefore, the suppression of the $\alpha$-fiber, i.e., the component which induces a higher thermal expansion coeffcient, during recrystallization could produce a decrease of the overall thermal expansion coefficient. Thus, a slope change in the dilatometry curve could be recorded. Such a change should be more pronounced when the intensity of the $\alpha$-fiber previous to recrystallization is more intense, i.e., in the sample with a higher degree of cold rolling reduction. This is consistent with the experimental results presented in this work.

\section{Conclusions}

The rearrangement and annihilation of dislocations associated with the recovery and recrystallization process and the simultaneous reorientation of the ferrite lattice will both induce the recorded slope change previous to the well known ferrite-to-austenite transformation detected during continuous heating.

\section{Acknowledgements}

The authors acknowledge financial support from the European Union through the European Coal and Steel Community programme (ECSC-7210-PR-368) and from the Spanish Ministerio de Ciencia y Tecnología (Special Action MAT 2002-10810-E). F.G. Caballero would like to thank the Spanish Ministerio de Educación y Ciencia for the financial support in the form of a Ramón y Cajal contract (Programa RyC 2002). T. De Cock would also like to express his gratitude to the Consejo Superior de Investigaciones Científicas (CSIC) for financial support in the form of a PhD research grant (I3P program). 


\section{References}

[1] Bunge HJ. Direccional Properties of Materials, Bunge HJ: DGM Informationsgesellchaft mbH; 1988. 1.

[2] Hutchinson W. Intern Mat Rev 1984; 29:25.

[3] Bethe K, Hölscher M, Lücke K. Proc 10th Int Conf On Texture of Materials ICOTOM'10. Bunge HJ: Clausthal, Germany; 1993. p.302.

[4] Raabe D, Lücke K. Mat Sci Technol 1993; 9:302.

[5] Klinkenberg C, Raabe D and Lücke K. Steel Research 1994; 65:291.

[6] Han HN, Lee JK and Kim S-J. Mat Letters 2005; 59:158.

[7] Prokoshkin SD, Khmelevskaya IY, Khmelevskii AV, Korotitskii AV, Zel'dovich VI, Khundzhua AG and Chumlyakov YI. The Physics of Metals and Metallography 2004; 6:596.

[8] MacDonald RA. Termal Expansion-8. Hanhn TA, Plenum Press, New York, USA; 1984. p.11.

[9] Rao KVK. Termal Expansion-1973 vol. 17, Taylor RE and Denman GL: AIP Conf Proc, New York, USA; 1974. p. 219.

[10] Megaw HD. Mater Res Bull 1971; 6:1007.

[11] Perez-Prado MT, Ibañez J, Morris M, Cristina MC, Ruano OA and Gonzalez-Doncel G. Rev Metal Madrid 1998;34:324.

[12] Bunge HJ. Texture Analysis in Materials Science. Bunge HJ:Butterworths, London, UK; 1982. p.1.

[13] García de Andrés C, Caruana G and Alvarez LF. Mater Si Eng 1998; A241:211.

[14] Lepera FS. Journ of Metals 1980; 32:38.

[15] Inagaki H. ISIJ Int 1994; 34:313.

[16] Barnet MR, Jonas JJ and Hodgson PD. 31th MWSP Conf Proc. Hamilton, Ontario, Canada: Iron and Steel Society; 1996. p.971.

[17] Ushioda K and Hutchinson WB. ISIJ Int 1989; 29: 862.

[18] García de Andrés C, Caballero FG and Capdevila C. Scripta Materialia 1998; 38:1835.

[19] García de Andrés C, Caballero FG and Capdevila C, Rev Metall Madrid 1998; 34:243.

[20] Speich GR, Demarest VA, Miller RL. Metall Trans A 1981; 12:1419.

[21] Stehle H and Seeger A. Z:Physik, 1956;146:217.

[22] Lomer WM. Phil Mag 1957; 2:1053.

[23] Hertzberg RW. Deformation and Fracture Mechanics of Engineering Materials, 3rd ed., John Wiley \& Sons, Inc., New York, NY: 1989. p.13.

[24] Timoshenko SP and Goodier JN. The Theory of Elasticity, $3^{\text {rd }}$ ed., McGraw-Hill, New York, USA: 1970. 